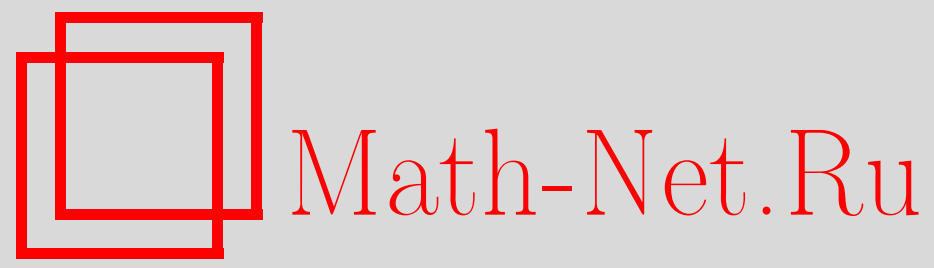

Ю. В. Мощенский, Метод моделей в дифференциальном термическом анализе, Вестн. Сам. гос. техн. ун-та. Сер. Физ.-мат. науки, 2001, выпуск 12, 150-156

DOI: https://doi.org/10.14498/vsgtu76

Использование Общероссийского математического портала Math-Net.Ru подразумевает, что вы прочитали и согласны с пользовательским соглашением

http://www.mathnet.ru/rus/agreement

Параметры загрузки:

IP : 52.90 .164 .192

26 апреля 2023 г., $15: 13: 29$ 


\title{
Термодинамика
}

\author{
УДК 543.226
}

Ю.В.Мощенский

\section{МЕТОД МОДЕЛЕЙ В ДИФФЕРЕНЦИАЛЬНОМ ТЕРМИЧЕСКОМ АНАЛИЗЕ}

\begin{abstract}
Для повышения точности и разрешающей способности при измерении малых количеств теплоты 6 устройствах дифференциильного термического анализа предлагается применить метод моделей. Анализируется схема измерений как система автоматического регулирования, охваченная глубокой отрицательной обратной связью.
\end{abstract}

Метод дифференциального термического анализа (ДТА) является наиболее распространённым среди термических методов анализа веществ вследствие простоты технической реализации. Однако платой за эту простоту является низкая точность измерений теплоты фазовых переходов (ФП) веществ и разрешающая способность по температуре. Указанное обстоятельство затрудняет исследование кинетики процессов химических и физических превращений в веществах, необходимое при анализе имеющихся и синтезе новых веществ и материалов. Поэтому актуальной является проблема повышения точности измерений с помощью простой и доступной аппаратуры ДТА.

В работе [1] построена модель теплообмена в «идеальной» и «реальной» системе ДТА, позволившая выявить нелинейность зависимости между количеством теплоты, выделившейся в процессе ФП, и площадью зарегистрированного температурного пика кривой ДТА. Также подтверждён тот факт, что коэффициент преобразования К термоаналитической ячейки (ТАЯ), как первичного измерительного преобразователя, является температурозависимым и эта зависимость в самом общем виде может быть выражена полиномом четвёртой степени от температуры в печи. Нелинейный характер зависимости характеристик температурного пика от теплоты ФП при использовании характерной для ДТА линейной аппроксимации типа $\Delta H m=K S$, где $\Delta H$-удельная теплота ФП; $\mathrm{S}$ - площадь температурного пика, является источником методической погрешности измерений.

Экспериментальные температурные кривые, регистрируемые устройствами ДТА, не являются истинными в смысле отображения исследуемого процесса тепловыделения во времени. Наличие термозависимого сопротивления между исследуемым образцом вещества и датчиком температуры приводит к «смазыванию» экспериментальной кривой, которое влияет на все измеряемые величины. Таким образом, имеет место следующая задача: рассчитать из экспериментальной кривой параметры неизвестного сложного процесса. Такая задача в настоящее время решается двумя методами: преобразованием Фурье и рекурсивным методом. Оба метода требуют численных расчётов на ЭВМ и для современных вычислительных средств проблемы не представляют. Рекурсивный метод широко применяется в калориметрии и даёт кривые теплового потока с хорошим разрешением и высокой точностью при условии линейной и хорошо воспроизводимой работы приборов [2]. В устройствах ДТА требование линейности не выполняется, сами измерения являются косвенными, т.к. искомое значение величины количества теплоты ФП определяется на основании некоторых соотношений, связывающих эту величину и результаты измерений температур (которые при использовании термопар также являются косвенными). Таким образом, в них фактически реализуется метод непосредственной оценки, когда осуществляется несколько последовательных преобразований сигнала измерительной информации в одном направлении (т.е. без обратной связи). Сравнение измеряемой величины с единицей измерения осуществляется косвенно путём предварительной калибровки. Систематическая составляющая погрешности может быть снижена различными видами калибровок, причём снижение будет тем значительнее, чем точнее совпадение всех теплофизических параметров калибровочного ( реперного ) вещества с исследуемым образцом. Случайную составляющую погрешностей в условиях изменения во времени измеряемой величины уменьшить значительно сложнее [3]. Калибровочные эксперименты необходимо производить при любых изменениях условий измерений, а, поскольку термоаналитическое исследование - это сложный 
многофакторный эксперимент, причём «многие факторы изменяются независимо по неустановленным законам» [4], то обязательно будут различия между измерительным и калибровочным экспериментом, приводящие к появлению значительных погрешностей измерений. Поэтому, несмотря на широкое внедрение вычислительных средств в процесс измерения и обработки результатов, проблема комплексного повышения точности и разрешающей способности устройств ДТА при условии сохранения простой конструкции ТАЯ не может быть решена без применения методов, основанных на введении структурной избыточности.

1 Выбор метода измерений. При измерении различных электрических и неэлектрических величин широкое распространение получили методы сравнения. На этих методах основаны мостовые, компенсационные и дифференциальные измерительные цепи. Метод сравнения предусматривает сопоставление параметров объекта измерения с известными параметрами образцового элемента и установление однозначного соответствия между ними. Этот принцип можно применить и к исследованию тепловых процессов. Частным случаем метода сравнений является метод моделей (рис.1.). Модель объекта должна быть регулируемой, а прибор сравнения выполнять функции индикатора равенства между собой измеряемой величины и меры. Эффект повышения точности результатов измерений будет тем значительнее, чем ближе значение меры к истинному значению измеряемой величины. В пределе метод превращается в нулевой [5], обеспечивающий высокую точность измерений.

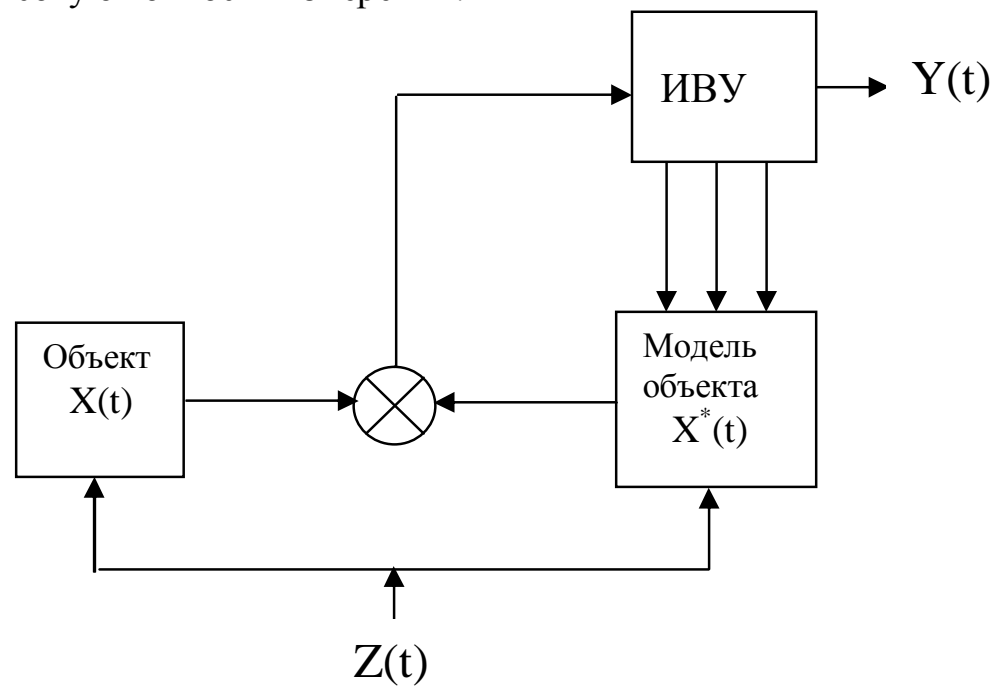

Р и с. 1. Схема измерений по методу моделей

Поскольку сравнение измеряемой величины производится автоматически, измерительный прибор имеет цепь отрицательной обратной связи, что иллюстрирует рис.2., на котором оригиналы функций $X(t)$ и $X^{*}(t)$ для удобства дальнейшего анализа заменены изображениями по Лапласу.

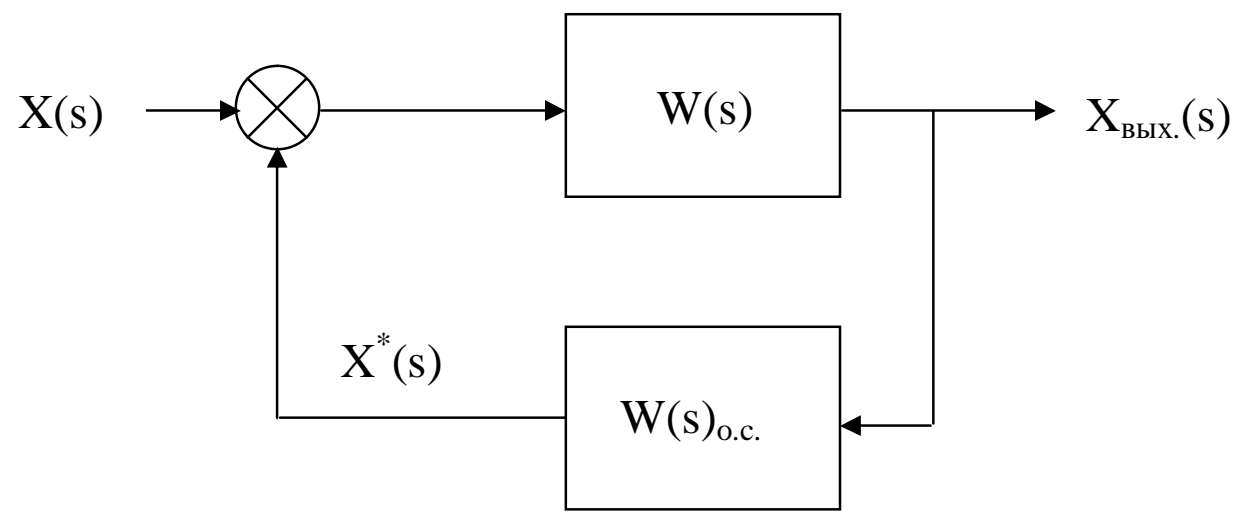

Р и с. 2. Схема измерений с использованием обратной связи

Следовательно, общая передаточная функция выразится формулой

$$
W(s)=\frac{W(s)}{1+W(s) W_{\text {o.c. }}(s)} \text {. }
$$


Для дальнейшего анализа преобразуем структурную схему измерительной системы (ИС) в более детальную (рис.3).

Здесь часть устройства, заключенная в пунктирную рамку, представляет из себя следящую систему, задача которой - отслеживание выходной величиной $X_{b b x} .(t)$ входной величины $X_{6 x} .(t)$, которая представляет собой изучаемый тепловой процесс. Аналитическое выражение для динамической характеристики измерительного устройства можно найти, используя зависимости, широко известные в технике регулирования [6]:

$$
W(s) X_{\text {bblx. }}=\frac{W_{1}(s) W_{2}(s)}{1+W_{4}(s) W_{3}(s) W_{2}(s)} .
$$

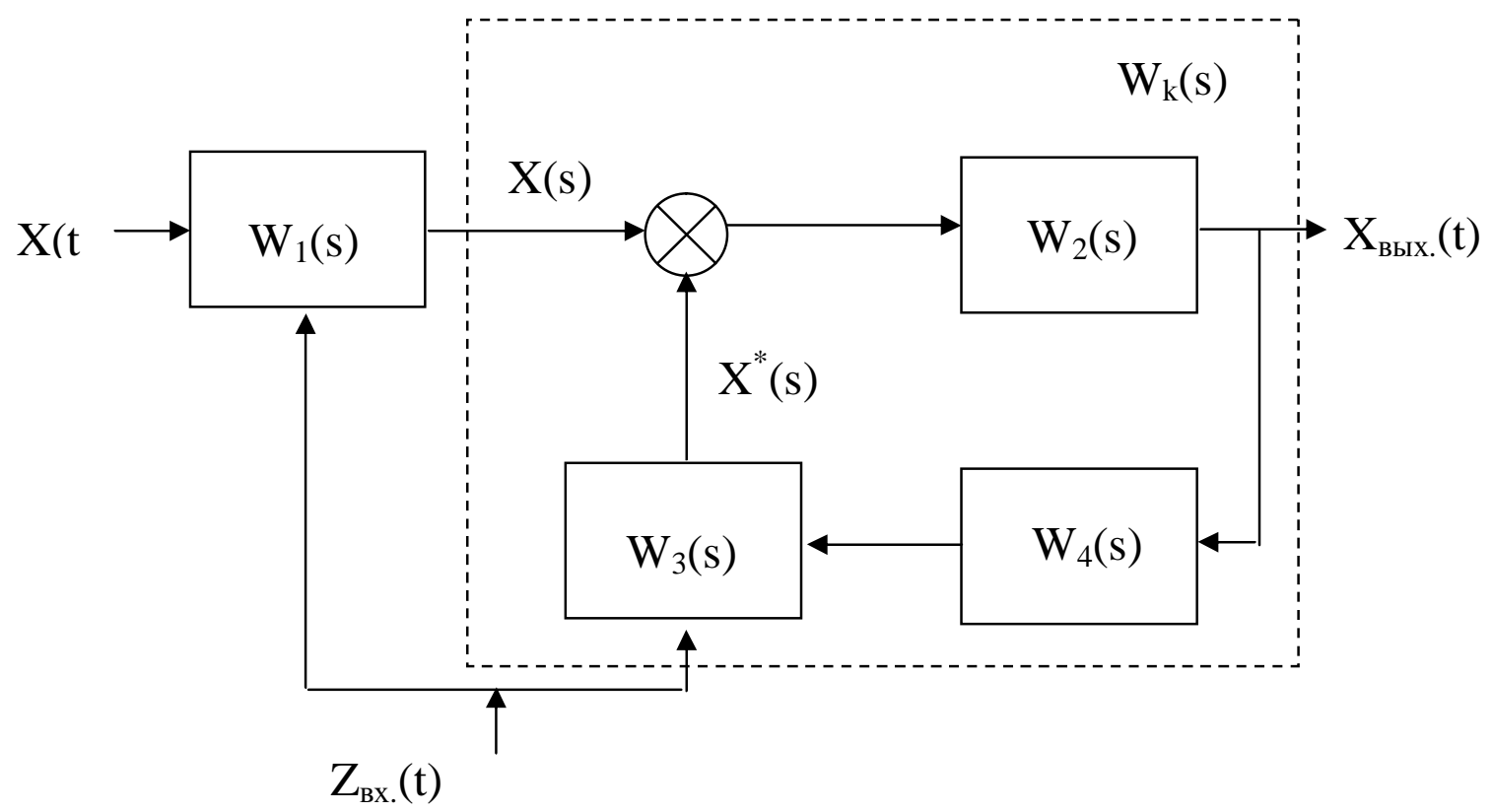

Р и с. 3. Укрупнённая схема измерений с обратной связью

Первичный преобразователь - термоаналитическую ячейку - можно представить в виде апериодического звена первого порядка с передаточной функцией

$$
W_{1}(s)=\frac{K_{1}}{1+\tau_{1} s} .
$$

Передаточная функция $W_{3}(s)$ имеет ту же характеристику, поскольку звено $W_{3}(s)$ представляет собой имитационную ячейку, в которой моделируется исследуемый тепловой процесс и конструктивно должны быть обеспечены условия максимального теплофизического подобия измерительной ТАЯ, т.е.

$$
W_{3}(s)=\frac{K_{3}}{1+\tau_{3} s} .
$$

Если пренебречь постоянной времени регулятора $W_{2}$, представляющего собой усилитель, то

$$
W_{2}(s)=K_{2} .
$$

На основании (1) передаточную функцию всей системы можно представить в виде

$$
W_{X_{B b L} .}(s)=\frac{K_{1} K_{2}}{\left(1+\tau_{1} s\right)\left(1+\frac{K_{2} K_{3} W_{4}(s)}{1+\tau_{2} s}\right.} .
$$

Если предположить, что $\tau_{1}=\tau_{2}$, то

$$
W_{X_{B L X .}}(s)=\frac{K_{1} K_{2}}{1+\tau s+K_{2} K_{3} W_{4}(s)}=K_{1}^{*} K_{2}^{*} \frac{1}{1+K_{2} \tau s}
$$

где $K_{1}^{*}=K_{1} K_{2}, K_{2}^{*}=\left(1+W_{4}(s) K_{2} K_{3}\right)^{-1}$

Постоянная времени $\tau=K_{2}^{*} \tau_{1}$ в $K_{2}^{*}$ раз меньше, чем у нескорректированной системы, причем для $K \rightarrow \infty \tau \rightarrow 0$. 
2 Оценка результирующей погрешности измерений количества теплоты. Измерение количеств теплоты ФП веществ при помощи разрабатываемой ИС осуществляется при помощи автоматического моделирования исследуемых процессов тепловыделения в имитационных ячейках при помощи встроенных микронагревателей с последующим преобразованием и обработкой данных измерений. Погрешности возникают на различных этапах измерений и обработки данных. Для их оценки необходимо исследовать природу возникновения и накопления погрешностей.

Основные составляющие погрешности ИС можно разбить на две группы:

-методическая погрешность;

-инструментальная погрешность.

Для получения количественных значений данных погрешностей необходимо установить закономерности возникновения и накопления их в процессе получения и обработки информации. Методическая погрешность состоит из погрешности адекватности модели и погрешности, связанной с особенностями обработки экспериментальных данных. Погрешность, связанная с особенностями обработки экспериментальных данных, состоит из двух составляющих:

-погрешности, связанной с алгоритмом собственно интегрирования площади термоаналитического пика (пренебрежимо мала, поэтому не рассматриваем);

-погрешности, связанной с алгоритмом ограничения площади пика.

Аналитическая оценка величины погрешности, связанной с алгоритмом ограничения площади пика, подлежащей интегрированию, крайне затруднена, поскольку "Вопрос о том, какая часть площади пика соответствует тепловому эффекту, является дискуссионным до сих пор" [7]. Анализ большого количества литературных источников позволил автору выделить до 15 вариантов ограничений, предложенных и обоснованных как теоретически, так и экспериментально. Поэтому для обоснования выбора способа ограничения площади термоаналитического пик был проделан большой объем экспериментальных исследований погрешностей ИС. В результате проделанной работы была подтверждена гипотеза об адекватности полной площади пика величине теплового эффекта фазового перехода для данной ИС.

Погрешность адекватности модели связана с особенностями технической реализации метода имитации тепловых процессов, происходящих в исследуемом веществе, а именно, с воспроизведением тепловых процессов, протекающих в исследуемом веществе, при помощи электрического микронагревателя. В случае экзотермического процесса разность температур между образцом и печью (температура печи растет по линейному закону) уменьшается по сравнению с предшествующим квазистационарным состоянием, вследствие чего тепловой поток от стенок печи к держателю образца (пропорциональный разности температур) также уменьшается и температура образца растет медленнее до тех пор, пока через некоторый интервал времени не установится прежнее тепловое состояние. При эндотермическом фазовом переходе температура вещества не будет изменяться до тех пор, пока не закончится процесс; тепловой поток между держателем образца и печью увеличивается вследствие повышения разности температур. В момент окончания фазового перехода разность температур превышает таковую при квазистационарных условиях. Это вызывает больший тепловой поток до тех пор, пока не восстановятся начальные условия.

Методическая погрешность возникает также вследствие различий условий теплообмена с печным пространством измерительных и имитационных ячеек. Оценим ее величину. Разрабатываемая ИС представляет из себя систему с непрерывной автоматической имитацией исследуемого теплового процесса. Технически такая система реализована в виде четырехэлементного устройства ДТА [8], в котором к паре образец-эталон добавлена пара соответствующих имитаторов образца и эталона. Регулирование мощности тепловыделения в микронагревателях имитаторов организовано таким образом, чтобы обеспечивалось равенство попарно дифферен-

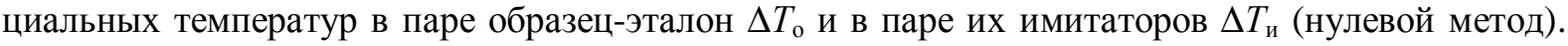
Можно показать (в рамках идеальной системы) что $\gamma=q$, где $q$ - мощность тепловыделения в имитаторе образца.

Теплообмен в реальной системе может быть описан с учетом возмущений параметров системы. Считаем, что теплота ФП равномерно распределена в интервале времени $\left[0, t_{\phi}\right]$ :

$$
\gamma=\frac{H \rho}{t_{\phi}}=\text { const }, \quad 0<t<t_{\phi} .
$$

Аналогичное предположение примем относительно мощности тепловыделения в имитаторе образца: 


$$
q=\frac{q_{o} \rho}{t_{\phi}}=\text { const }, \quad 0<t<t_{\phi},
$$

где $q_{\mathrm{o}}$ - удельная величина тепловыделения в имитаторе образца.

Если теперь потребовать равенства по модулю максимальных значений дифференциальных температур в паре образец-эталон и в паре имитаторов $\left|\Delta T_{\text {omax }}\right|=\left|\Delta T_{\text {иmax }}\right|$, то решение линеаризованной задачи теплообмена приводит к следующим расчетным зависимостям между $H$ и $q_{\mathrm{o}}$ :

$$
H=\left\{\begin{array}{cc}
q_{o}-\frac{t_{\phi} C^{\prime} \Phi}{\rho}, & H>0 ; \\
-q_{o}+\frac{t_{\phi} C^{\prime} \Phi}{\rho}, & H<0 .
\end{array}\right.
$$

Видно, что погрешность уменьшается с относительным уменьшением массы активного вещества $\left(\mathrm{t}_{\phi} \rightarrow 0\right)$.

Величина инструментальной погрешности устройства будет определяться, главным образом, качеством работы контура имитации исследуемых тепловых процессов, как автоматической следящей системы, поскольку такими составляющими инструментальной погрешности, как погрешность от градуировки термопар (не превышает $0,02 \%$ для диапазона температур $0 \ldots 1000^{\circ} \mathrm{C}$ ), погрешность от ограниченной разрядности АЦП (для примененных 12-ти разрядных АЦП не превышает 0,025\%), а также машинными можно пренебречь.

3 Оценка качества регулирования. Качество работы следящей системы контура моделирования тепловых процессов определяется величиной ошибки, равной разности между требуемым и действительным значениями регулируемой величины. Для определения качественных показателей используются критерии качества. Пусть $Z(t)$ - возмущающее воздействие на объект регулирования, а $X(t)$ - задающее воздействие (рис.3). Регулируемая величина $X^{*}(t)$ может быть найдена из выражения

$$
X^{*}(t)=W_{o}(s) X(t)+W_{z}(s) Z(t)
$$

где $W_{o}(s)$-передаточная функция регулируемого объекта по регулирующему воздействию; $W z(s)$-передаточная функция регулируемого объекта по возмущающему воздействию.

Рассмотрим случай, когда имеется только задающее воздействие $X(t)$. Если $X(t)$ имеет произвольную форму, но достаточно плавную, т.е. через некоторое время существенное значение имеет только конечное число $m$ производных (что всегда выполняется для рассматриваемых тепловых процессов), т.е.

$$
\frac{d x}{d t} ; \frac{d^{2} x}{d t^{2}} ; \mathrm{L} ; \frac{d^{m} x}{d t^{m}},
$$

то ошибку системы можно определить следующим образом:

$$
\Delta X(s)=\Phi_{x}(s) X(s)=\frac{X(s)}{1+W(s)}
$$

где $\Phi_{\mathrm{x}}(s)$ - передаточная функция замкнутой системы по ошибке; $X(s)$ - изображение задающего воздействия.

Разложим передаточную функцию по ошибке в выражении (6) в ряд по возрастающим степеням комплексной величины $s$ :

$$
\Delta X(s)=\left[C_{o}+C_{1} s+\frac{C_{2}}{2 !} s^{2}+\frac{C_{3}}{3 !} s^{3}+\mathrm{K}\right] X(s),
$$

сходящийся при малых значениях $s$, т.е. при достаточно больших значениях времени $t$, что соответствует установившемуся процессу изменения регулируемой величины при заданной форме управляющего воздействия. Переходя в (7) к оригиналу, получаем формулу для установившейся ошибки

$$
X_{y c m .}=C_{o} X(t)+C_{1} \frac{d X(t)}{d t}+\frac{C_{2}}{2 !} \frac{d_{2} X(t)}{d t^{2}}+\mathrm{K},
$$

где $C_{0}, C_{1}, C_{2}$ называются коэффициентами ошибок. Они могут быть определены согласно общему правилу разложения функции в ряд Тейлора по формулам: 


$$
C_{o}=\left[\Phi_{x}(s)\right]_{s=0} ; \quad C_{1}=\left[\frac{d \Phi_{x}(s)}{d s}\right]_{s=0} ; \mathrm{K} ; C_{m}=\left[\frac{d_{m} \Phi_{x}(s)}{d s^{m}}\right]_{s=0} .
$$

Передаточная функция по ошибке имеет вид

$$
\Phi_{x}(s)=\frac{1}{1+W(s)}=\frac{\tau_{3} \tau_{4} s^{3}+\left(\tau_{3}+\tau_{4}\right) s^{2}+s}{\tau_{3} \tau_{4} s^{3}+\left(\tau_{3}+\tau_{4}\right) s^{2}+s+K} .
$$

Деля числитель на знаменатель, получаем ряд

Сравнение этого ряда с (7) дает

$$
\Phi_{x}(s)=\frac{1}{K} s+\left(\frac{\tau_{3}+\tau_{4}}{K}-\frac{1}{K^{2}}\right) s^{2}+\left(\tau_{3} \tau_{4}-2 \frac{\tau_{3}+\tau_{4}}{K}+\frac{1}{K^{2}}\right) s^{3} .
$$

$$
C_{o}=0 ; \quad C_{1}=\frac{1}{K} ; \quad C_{2}=2\left(\frac{\tau_{3}+\tau_{4}}{K}-\frac{1}{K^{2}}\right) .
$$

Коэффициент $C o=0$ характеризует систему как астатическую, т.е. не имеющую ошибки статизма. Коэффициент $C_{1}$ называют скоростной ошибкой, равной отношению скорости задания к добротности системы по скорости; коэффициент $C_{2}$ представляет собой добавочную ошибку от постоянного ускорения и характеризует добротность системы по ускорению.

4 Оценка динамической погрешности. Из анализа математической модели следует, что и коэффициент преобразования $K$ и постоянная времени термоаналитической ячейки являются температурозависимыми величинами, поэтому условия корректировки должны распространяться на весь диапазон рабочих температур ИС, вследствие чего необходимо исследовать параметрическую чувствительность скорректированной ИС. Для удобства анализа преобразуем структурную схему (см. рис.2) в эквивалентную схему, представленную на рис.5.

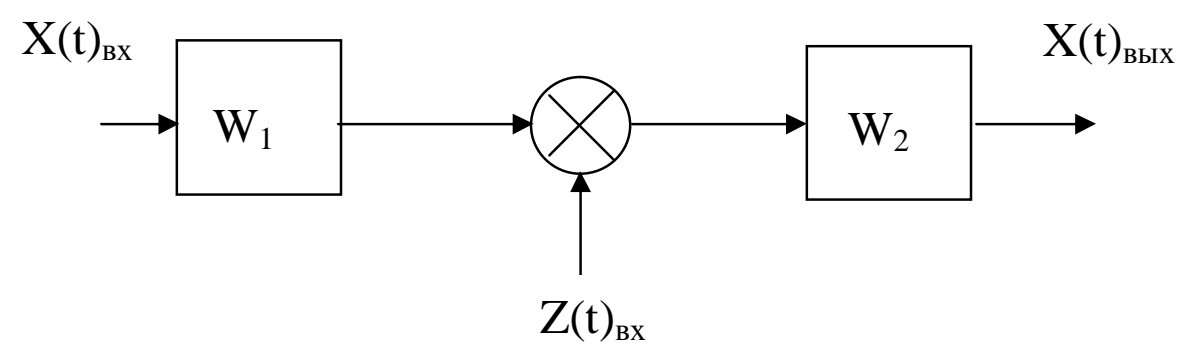

Р и с. 5. Эквивалентная схема измерений

Из выражения(2) для передаточной функции термоаналитической ячейки с постоянной времени

$$
\tau_{1}=\frac{C \rho v}{\Phi F_{H}}=\frac{C_{o} \rho_{o} v}{\Phi F_{H}}
$$

где $C_{o}$ - удельная теплоемкость, $\Phi$ - некоторая функция параметров теплообмена, находим выражение для граничной частоты $\omega g$ системы (при которой значение амплитудной характеристики $|W(j \omega)|$ уменьшается на 3 дБ, т.е. до $1 / \sqrt{ } 2$ от первоначальной величины):

$$
\omega_{g}=\frac{1}{\tau_{1}}, \text { или } f_{g}=\frac{1}{2 \pi \tau_{1}} .
$$

Будем считать, что из всех параметров сильно изменяется только коэффициент теплообмена $\Phi$, в то время как другие параметры остаются практически постоянными. При расчете коэффициента параметрического влияния для граничной частоты относительно параметра Ф запишем выражение

$$
v f_{g}=\frac{\partial}{\partial \Phi} \frac{\Phi F_{H}}{2 \pi C \rho v}=\frac{F}{2 \pi C \rho \nu} .
$$

Для коэффициента параметрического влияния времени установления режима относительно параметра Ф при времени установления $t_{\mathrm{e}} \approx 3 \tau_{1}$ получаем

$$
v \tau_{e}=\frac{\partial}{\partial \Phi} \frac{\pi C \rho v}{\Phi F_{H}}=-\frac{\pi C \rho v}{\Phi 2 F_{H}}
$$


Изменение параметра влечет за собой изменение постоянной времени на величину $\Delta \tau_{1}$, при этом, согласно уравнению (8), между изменением коэффициента теплообмена и изменением постоянной времени существует зависимость

$$
\frac{\Delta \tau_{1}}{\tau_{1}}=-\frac{\Delta \Phi}{\Phi_{o}}
$$

Вследствие того, что в общем случае идеально скорректировать систему не представляется возможным, т.е. $\tau_{2}=\tau_{1}+\Delta \tau$ получаем

$$
W_{\text {общ. }}(s)=\frac{C\left(1+\tau_{1} s+\Delta \tau s\right.}{\left(1+\tau_{1} s\right)\left(1+a \tau_{1} s+a \Delta \tau s\right)},
$$

где $a=\tau_{\kappa} / \tau_{1}$ - коэффициент уменьшения постоянной времени скорректированной системы.

Применяя к (13) преобразование Лапласа, получаем переходную функцию

$$
h(t)=\frac{C}{2 \pi j} \int_{\sigma-j \infty}^{\sigma+j \infty} \frac{W_{\text {общ. }}}{s} e^{s t}=C\left[1-e^{-\frac{t}{a\left(\tau_{1}+\Delta \tau\right)}}\right]+C \frac{\Delta \tau}{\tau_{1}+a\left(\tau_{1}+\Delta \tau\right)} e^{-\frac{t}{\tau_{1}}}-e^{-\frac{t}{a\left(\tau_{1}+\Delta \tau\right)}} .
$$

Анализ этого выражения показывает, что при отклонении постоянной времени от оптимальной появляется динамическая погрешность и переходные процессы могут значительно удлиняться во времени. Поэтому важнейшим условием исключения параметрического влияния изменяющихся параметров является достижение максимальной идентичности характеристик ячеек с образцом и имитационных ячеек по всему диапазону температур.

Экспериментальное исследование параметров опытного образца устройства [9] подтвердило существенно возросшие (на порядок) точность и разрешающую способность благодаря использованию моделирования исследуемого теплового процесса непосредственно в ходе эксперимента.

Выводы.

Широко используемое в калориметрии воспроизведение истинных кривых теплового потока с помощью преобразования Фурье и рекурсивного метода для устройств ДТА нецелесообразно вследствие невыполнения требования линейности.

В устройствах ДТА реализуется метод непосредственной оценки, когда осуществляется несколько последовательных преобразований сигнала измерительной информации без обратной связи, поэтому для повышения точности измерений целесообразно применение методов, основанных на структурной избыточности.

Для количественных измерений теплоты фазовых переходов веществ предложено применить частный случай метода сравнений - метод моделей, основанный на использовании автоматической следящей системы, задающим воздействием для которой является исследуемый тепловой процесс.

Преобладающей в инструментальной погрешности является погрешность работы контура моделирования исследуемого теплового процесса как следящей системы автоматического регулирования. Условием получения наивысшей точности измерений количеств теплоты и разрешающей способности по температуре является обеспечение максимальной идентичности характеристик ячеек с образцом и имитационных ячеек по всему диапазону рабочих температур.

\section{БИБЛОГРАФИЧЕСКИЙ СПИСОК}

1. Мощенский Ю.В. Информационно-измерительная система термического анализа : Дисс. ...канд. техн. наук // Самара, 1998, 83c.

2. Хеммингер В., Хеке Г. Калориметрия. Теория и практика. М.: Химия. 1990. 176с.

3. Куликовский К.Л., Купер В.Я. Методы и средства измерений. М.: Энергоатомиздат. 1986. 448c.

4. Шестак Я. Теория термического анализа. Физико-химические свойства твёрдых неорганических веществ. М.: Мир. 1987. 456c.

5. Краус М., Вошни Э. Измерительные информационные системы. М.: Мир. 1975. 463с.

6. Бесекерский В.А., Попов У.В. Теория систем автоматического регулирования. М.: Наука. 1975. 768c.

7. Измалков А.Н. Определение теплот реакций методом ДТА: Дисс. ...канд.хим. наук // Куйбышев, 1989. 180с

8. Авт. свид. №1376019 (СССР) Устройство для дифференциального термического анализа. / Мощенский Ю.В., Трунин А.С.

9. Мощенский Ю.В., Трунин А.С., Космынин А.С. Система термического анализа для калориметрических исследований. Самара: СамГТУ. 1999. 64c. 